\title{
A Cognitive Approach to Language-force of Chongqing Dialect Particle_-A Case Study of "ma"
}

\author{
Liyao Tang \\ College of Foreign Languages and Literatures, Chongqing Normal University, China \\ Lian Xiong \\ College of Foreign Languages and Literatures, Chongqing Normal University, China
}

\begin{abstract}
Modal particle is an important mean of expressing inner emotion in daily communication, and it plays an irreplaceable role in language. Chongqing dialect, as the essence of the local culture of china, should be inherited eternally. This research focuses on the study of the modal word of "ma" in the dialect of Chongqing and analyzes its cognitive process and characterization of the word in human brain under the guidance of Tamly's force - dynamic model, acquiring the functions and effects of "ma" in different contexts which are expected to promote the development and preservation of local culture in Chongqing and provide a reference for dealing with interpersonal relationship.
\end{abstract}

Index Terms - Chongqing dialect, ma, force dynamics theory

\section{INTRODUCTION}

Chongqing dialect is also called Chongqing idiom by native speakers with the features of hard, straight and plane. During the Ming Dynasty, many people from Hunan settled here, accounting for $40 \%$ of the total population of Chongqing, but Chongqing dialect is completely preserved with its strong vitality.

Recently, there are some researches about dialect at home and abroad, but most of which just study from aspects of translation of dialect, its pronunciation system, or the effects on the acquisition of other languages of learners. Brady (2015) has figured out that dialect is not only important for the native adults, but also the teenagers. His paper explores the impact between adolescent identity and standard English or dialect from a political perspective. By conducting an experiment on 54 students in London, his paper works out that dialect plays an important role in constructing teenagers' identities. What's more, Han Ziman (2002) discusses the limitations of dialects in translating foreign literary works into Chinese. He insists dialects in different countries have different characteristics. It can not be expressed equally in translation so that he provides methods of using exoteric expression or filling-up after the text. Le Meiyun and Ling Dexiang (1994) explore the basic discipline of dialectical pronunciation in various regions of China and get the reason why speakers make such mistakes in pronouncing ---because there is an interference between dialect and English. Different dialects result in the situation of making different mistakes in pronunciation. Liu Juhong (1998) discusses the influence of Tibetan students' dialectical system to the acquisition of English. Just as the research of Le Meiyun, dialect is powerful enough to change the pronunciation of English. In the same way, it has an impact on the second language acquisition. As for the particle, a minority of scholars have discussed it before. Those studies have focused on its function, semantic conveying, hierarchical status or characteristics of different regional dialects or pragmatic values. Mrayat (2015) gives a description about the particles and morphemes with negative meanings in Jordanian Arabic. Zhu Dexi (1983) proposes to discuss a number of marked particles in the view of grammar and semantic function. Huang Guoying (1994) explains the levels of particles which are located at the end of the sentence, which means to compare the emotion-force of different particles. Fang Mei (1994) derives the division between primary and secondary information in a sentence which is reflected in the process of pronouncing the particle in a stressed or light tone. Nevertheless, most of them only concerned about syntactic or semantic effects with little concentration on emotion expressing.

In addition, many scholars have done various investigations to Chongqing dialect. Ming Maoxiu (2016) has conducted an experiment about tone in Chongqing dialect. In his study, there are some other tones which have not been found before by observing speakers' gender; Dong Sicong (2013) has researched on the negative words of Chongqing dialect. He draws a conclusion that there are some similarities and differences by comparing dialect with mandarin. Wang Changxue (1996) has devoted himself to the discussion of a certain voice--- "er" in Chongqing dialect. What's more, Han Weiwei (2013), Mao Lingshuang (2013),Dai Wei and Zhou Wende (1999) all have studied on the pronunciation of Chongqing dialect. However, most of which just study from aspects of translation of dialect, its pronunciation system, or the effects on the acquisition of other languages of learners. Few researches are carried on 
modal particles, especially in the dialect of Chongqing. Particle, as an important way to describe inner feelings, is often used in daily conversation. This article aims to explore the presentation, construction methods and the influence of language in human psychology under the guidance of Force Dynamic Model Theory and infers the production and consequence of speech, which may improve interpersonal communications and make our society more harmonious .

\section{The APPLiCATION OF FORCE DyNAMIC THEORY}

The traditional researches on "force" mainly focus on physics, energetics and other disciplines. There is few application in language. A famous cognitive linguist Leonard Talmy (1988) firstly proposes Force-Dynamic Model Theory. He insists that Force Dynamics--a mode of construing the world in terms of entities interacting with respect to force --is a neglected semantic category. As a result, he proposes to construct the meaning of language by means of "force" which can represent our kinesthetic system and demonstrate the universality of force dynamic thought.

According to Tamly's opinion, there are two basic elements in "force" unit: the Agonist and the Antagonist. Ungerer \& Schemid (1996) suggest that the perceptual field is always divided into figure and ground. The figure refers to the part which is highlighted. Therefore, the agonist as a focus is usually highlighted by foregrounding while the antagonist refers to the force which against the agonist. With time goes by, force dynamics begins to develop it into cross fields, especially the combination with psychology. Tamly (1995) argues that there is a great similarity between cognitive linguistics and psychology because of metaphor. Metaphor is firstly proposed by Lakoff and Johnson (1980). Later, Lakoff (1993) reckons that metaphor comes from thought rather than language. Generally speaking, when people describe a situation, they will construct the situation in mind (Langacker, 1987). What's more, Lakoff (1987) proposes that metaphor is the way of mapping from source domain to target domain which means to find out some same features between source and target domain. Zhao Yanfang (2001) explains that the mapping behavior refers not only to the expression in language, but also the way of thinking one thing to replace another related thing. Thus, force dynamic theory expands its application from metaphors of physics to psychology and sociology. It mainly refers to the similarity of psychological association and emotion caused by an entity involving in the psychological level, or a kind of binding force caused by the social responsibility or obligations. For example:

(1) She refrained from responding.

(2) He stayed even though they asked him to leave.

Example (1) reflects the split-self model which presents the state of mental activity can be active or static. The agonist force is she while the antagonist is herself in this situation. The force in example (2) is the interaction of society. The agonist is he while the antagonist is they. They have imposed a force to him to ask him to leave. According to the word stay, we know that the force of antagonist is less than the agonist at this moment so that the state of he can not be changed. Tamly (2000) indicates that there are four basic dynamic models in the force-dynamic model, which can explain the meaning of language effectively. The modal particle of Chongqing dialect plays an important role in expressing human emotions. Peng Yongzhao (1988) has made a systematic summary of several typical modal words in Chongqing dialect. Nevertheless, he has not made any academic contributions. Therefore, this paper will analyze the effect and validity of the modal words in Chongqing dialect under the guidance of Force Dynamic Theory.

\section{The LANGUAGe-FORCE OF “MA” IN ChOngQING DialeCt}

There are a lot of modal particles in Chongqing dialect, and one particle may have different functions. According to the systematic analysis of Chongqing dialect and the collected corpus (this corpus derives from the dialect program in Chongqing called Foggy Night), this paper finally chooses the word "ma" as the object to analyze the language-force of "ma" in sentences.

\section{A. The Force Balance of " $m a$ " in Application}

In the course of speech act, the speaker usually wants to change the state of the listener, while the hearer needs to respond according to his own situation. Therefore, There must exist an output of some feelings or needs when people say something. This chapter mainly discusses the force balance of "ma" from the perspective of two communicators.

Different particles have different functions in sentences, and even the same particle has different functions in different sentences. For example:

(1) Reminding:

a. Please remember to close the door.

b. You remember to close door yo.

c. Ni jide guan men yo.

(English version)

(Chinglish version)

(Chinese version)

(2) Surprising:

a. Oh, you have new clothes today.

b. Ye, you today wear new clothes o.

c. Ye, ni jintian chuan de xin yifu o.

(English version)

(Chinglish version)

(Chinese version)

(3) Easing tone:

a. Please remember to hand in your homework. (English version) 
b. Everyone remembers to hand in homework ha.

c. Dajia jide jiao zuoye ha.

(Chinglish version)

(Chinese version)

According to the examples above, we know that no matter what functions they want to realize, the expressions which include particles play the role of transferring emotions between speakers.

Therefore, "ma" as a typical particle in Chongqing dialect, also includes an emotional output in communication. It will be demonstrated in following examples:

(4) Strengthening tone:

a. You should be obedient.

(English version)

b. You gai obedient ma.

c. Ni shi gai tinghua ma.

(Chinglish version)

(Chinese version)

(5) Easing tone:

a. Hurry up.

b. Go fast ma.

c. Zou kuaidian ma.

(English version)

(Chinglish version)

(Chinese version)

Since the modal words aim to express our feelings, it is absolutely important why we choose to show this feeling and why we use such a particle. Wen Xu (2014) points out that the focus is always protruded by word orders and syntax structures, which is usually located at the end of the sentence. Combining with this article, we infer that the particle which is often at the end of sentence may also have the function of focus. "Ma" is used at the end of example (4) and (5). Hence one can see that the focus of the sentence has also been shifted to the end. Wardhaugh (1986) shows that language can not be separated from society. The production of speech acts must comply with the social norms. So both sides of communication are inevitably subject to social norms being in the social environment. For instance, in example (4), "whether you should be obedient or not" is subject to the social norms. For the listener, if he is young or he has done something wrong, he should be obedient. Meanwhile, the word "ma" is added to the end of the sentence to strengthen the speaker's emotional expression and this request (to be obedient) becomes naturally. So the hearer must obey the conditions to achieve the balance between speaker and listener and then to finish the purpose of communication. It is often difficult for people to accept sharp language or ignore some requirements caused by social environment, which also illustrates the reason why the above examples use the words "yo" "o" or "ma". In example (5), the same word "ma" plays another function of easing tone. Suppose to remove "ma", the sentence is an imperative sentence with strong emotion of ordering. So it is easy to make listeners rebellious, and difficult to obey orders. According to the principle of cooperation proposed by Grice (1975), speakers often use the method of adding the word "ma" in order to ease the tone and reduce the resistance of such a request in the mind of hearer, which can form an emotional balance between speakers and listeners and achieve the purpose of communication.

Form the above analysis, the correct use of particle in Chongqing dialect should mainly be based on the different perspectives of both sides of communication, taking the purpose and cognitive emotion of each other into accounts, and then the balance between listeners and speakers will be achieved.

\section{B. The Validity of "ma” Based on Force Dynamics}

In the expressions with particles, the dynamic balance between the discourse force of the speaker and the inner emotion force of the listener will be achieved. Mo Chiyang and Duan Yun (2012) have argued that the speaker tries to put a force to listener by using discourse to overcome hearer's inner force and change his state in communication. The force which changes hearer's state is called "language-force". Similarly, we can establish a language-force model of "ma" based on force-dynamic model system, which is mainly used to analyze the balance between the language-force of "ma" and the force of social psychological confrontation. According to the principle of force dynamic theory ---antagonist puts a force to agonist to change agonist's state---agonist is listener's will (including the inner thought, habits or the mood of hearers), and the antagonist is the language-force against listener (including the intention of the speaker, social customs, public psychological trends, interests, attitude to life, emotional courtesy, etc.) in the language-force model of "ma". This chapter mainly analyzes the validity of "ma" in application.

According to the statistical analysis of the corpora containing the word "ma", the validity of "ma" in application can be roughly divided into the following three categories:

(1) The function of strengthening tone:

"Ma" can be placed at the end of declarative sentence, indicating the attitude of "it should be" of the discourse, which plays the role of enhancing tone. Under the circumstance of force dynamics, the meaning of speaker's discourse can be consolidated. The language-force increases, so the effect is also enhanced in the dynamic system. For example:

(6) a. You should be obedient.

(English version)

b. You gai obedient ma.

c. Ni shi gai tinghua ma.

(Chinglish version)

(Chinese version)

(7) a. Thirties people are old enough to marry. (English version)

b. Dou thirties people should marry ma. (Chinglish version)

c. Dou sanshi hao ji de ren le gai jiehun le ma. (Chinese version)

According to the above analysis, the production of speech act in Example (6) is based on the fact that the speaker hopes the listener to be obedient. So the agonist (Fago) is the inner strength of the hearer (for a certain reason, he is 
insubordinate), while the antagonist (Fant) is the combination of the speaker's intention F1( the speaker hopes hearer to be obedient) and the social cultural factors F2 (the identity or status of the hearer are considered to be obedient in public thoughts) (Figure 1).

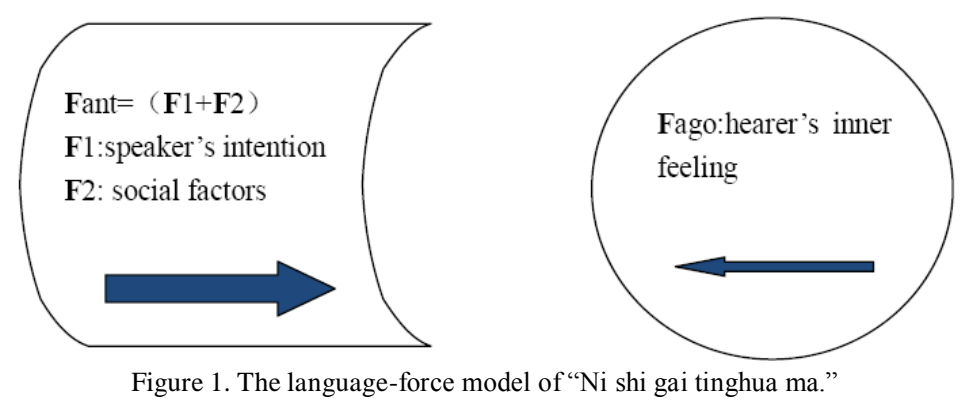

With the particle "ma" in the end, the emotion of the sentence increases. It means that Fant is enhanced and then Fago is relatively reduced. The forces of both sides are imbalance so that the language - force model tends to be dynamic. The whole force drives towards the right. That is to say, Fago is pushed by Fant. So we can say the particle "ma" is more effective in this case.

Similarly, "ma" in Example (7) plays the same role of enhancing the tone. In the dynamic balance system, agonist is the hearer's inner feeling of fearing or worrying, so he is reluctant to marry. The antagonist is the purpose of hoping hearer to get married from his parents or friends (F1) and the social trend that a person over thirty is old enough to marry (F2). By using "ma" to enhance the speaker's language-force, the possibility of constraining agonist and changing its state also increases (shown in Figure 2).
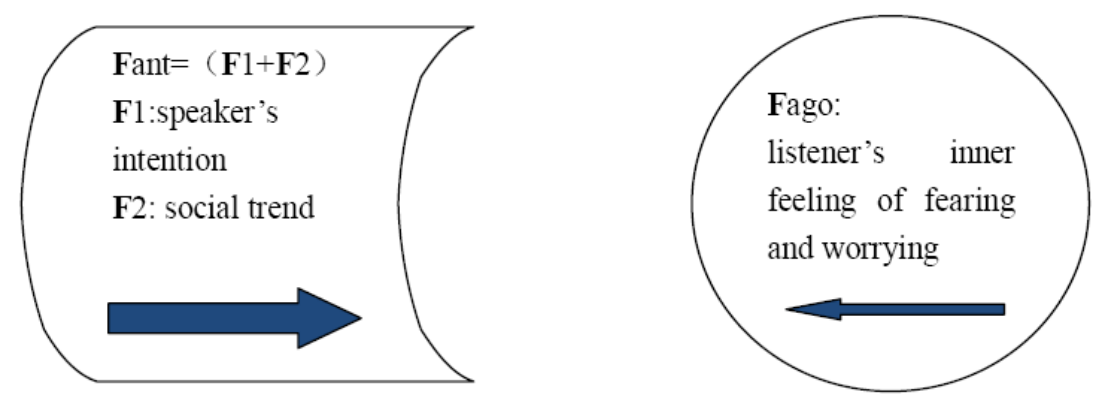

Figure 2. The language-force model of "Dou sanshi hao ji de ren le gai jiehun le ma."

(2) The function of easing tone:

"Ma" is often used at the end of the imperative sentence, and sometimes it is applied in the structure of the "V. + ma $+\mathrm{V}$ ". (eg: ku sazi ma ku). It will reduce the mood of giving order or request and ease the atmosphere. In this situation, the language-force of speaker decreases. At the same time, the constraint on hearer's psychology reduces, too. Therefore, the possibility of producing some behaviors caused by discourses increases. For example:

(8) a. Hurry up. It is almost late.

b. Go quickly ma. Yao late le.

(English version)

c. Zou kuaidian ma, yao chidao le.

(Chinglish version)

(9) a. Please be quiet.

(Chinese version)

b. You lower voice ma.

c. Ni xiao sheng dian ma.

(English version)

(Chinglish version)

(Chinese version)

Comparing with "zou kuaidian" or "ni xiao sheng dian", Example (8) and (9) are easier to accept. "Ma" in these sentences plays the role of easing tone. According to the analysis of the force-dynamic system, the agonist (Fago) in example (8) is the hearer's state, which may be exhausted or has other physical deficiency and he proceeds relatively slowly. The antagonist (Fant) is the combination of the speaker's intention that he does not want to be late(F1) and the social norm (F2) that the social does not advocate the habit of late. In example (9), the agonist is the the hearer's habit (Fago) that speaks loudly without intention. The antagonist is the force (Fant) that the speaker's intention of wanting a quiet environment (F1) and the social norm (F2) that we should keep quiet in public. There is a "ma" at the end of both sentences, which plays the role of easing tone. The force of imperative sentence is reduced, and the friction on hearer's reduced accordingly. Thereby, the possibility of accepting the advice is increased. From the perspective, "ma" plays an very important role in the force-dynamic system. However, if the word "ma" is deleted, the sentence has a very strong order. It impresses the hearer much rejection which may difficult to change the state of listener, and even leave strong impact and destroy the original effect (Figure 3). 

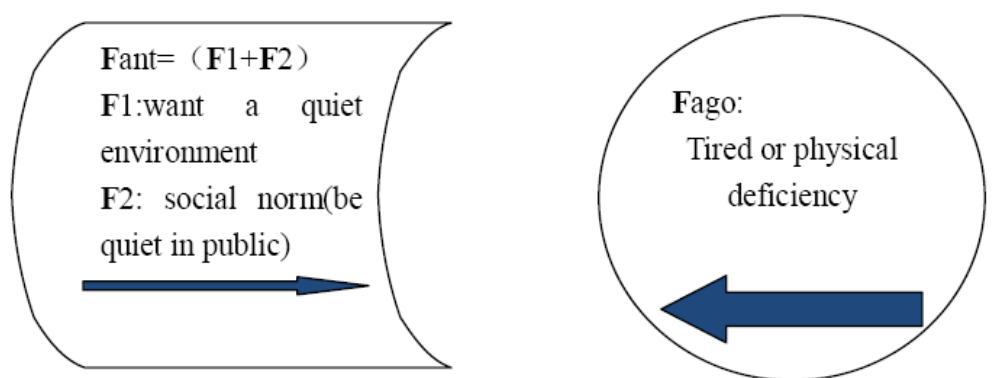

Figure 3. The language-force model of "Zou kuaidian ma, yao chidao le." or "Ni xiao sheng dian ma."

(3) The function of urging tone:

In this function, "ma" is often used at the end of the interrogative sentence, which urges the other side to respond quickly and highlights the speaker's psychology of thirsting for the answer. In dynamic force system, the context is given a sense of urgency, reflecting the anxiety of the speaker. For example:

(10) a. Do you like the clothes? (English version)

b. You like or not like the clothes ma?

(Chinglish version)

c. Ni xi bu xihuan zhege yifu ma?

(Chinese version)

(11) a. Are they?

b. Are or are not they ma?

(English version)

(Chinglish version)

c. Shi bushi zhe qun ren ma?

(Chinese version)

In Examples (10) and (11), the word "ma" effectively increases the interaction between the two communicators. Suppose that there is no "ma" in these two sentences. Then example (10) should be "Ni xi bu xihuan zhege yifu", which happens in general situation. If the listener did not answer the question, then the speaker can continue to ask, "Ni xi bu xihuan zhege yifu ma". The second expression is significantly increased the state of anxious. What's more, in example (10), the agonist (Fago) is the habit of the hearer or the current mood. The antagonist (Fant) is the combination of psychological state $(\mathbf{F} 1)$ of speaker of wanting to know the answer and social conditions (F2) (eg: Time is limited. They must decide to buy it or not quickly). The habit or emotion of listener is according to what he hears and then he gives response by quickly understanding the discourse in mind. Since the word "ma" itself has the urging function, the listener is more likely to make any responses in this context. Therefore, we may regard the word "ma" has worked effectively (Figure 4).
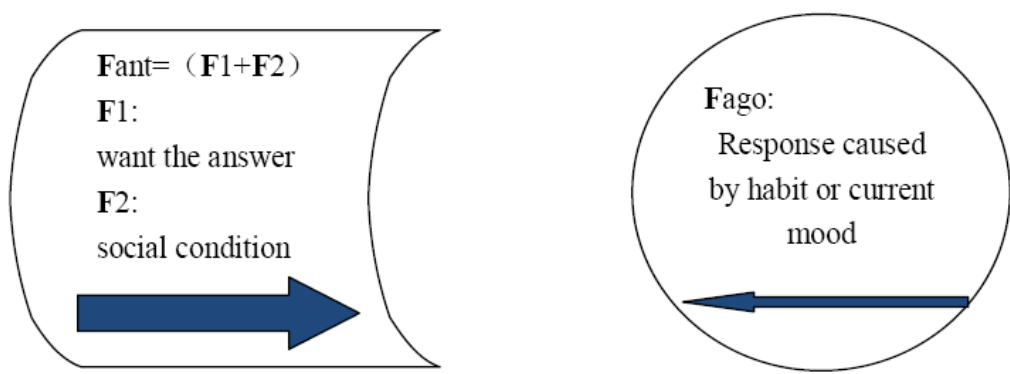

Figure 4. The language-force model of "Ni xi bu xihuan zhege yifu ma?"

\section{CONCLUSION}

This research analyzes the particle of "ma" in Chongqing dialect from the perspective of cognitive linguistics. From the perspective of both communicators and the Force-Dynamics Theory, the writer studies the characterization of "ma" in human cognitive system. A Language - dynamic system of "ma" is constructed according to Force Dynamic Theory. The particles of Chongqing dialect have an important role in guiding people to express their daily emotions. The corpora above which come from TV show are similar to our daily communication, and each expression may occur in different situations. Correspondingly, there may be different language - force models. However, there are still some shortcomings in the above analysis, so further investigations and inquiries are needed. It is hoped that this research can provide a reference for the daily communication of residents in Chongqing and offer broaden research ideas of studying dialects for other scholars.

\section{REFERENCES}

[1] Brady, J. (2015). Dialect, Power and Politics: Standard English and Adolescent Identities. Literacy, 49 (3), 149-157.

[2] Dai Wei \& Zhou Wende. (1999). The Phonetic Features of Chongqing Dialect. Journal of Chongqing Normal University, 01, 
98-102.

[3] Dong Sicong. (2013). Discourse Markings of Several Negative Words in Chongqing Dialect. Journal of Chongqing University of Posts and Telecommunications, 06, 120-124.

[4] Fang Mei. (1994). A Study on the Function of Modal Particles in Beijing Dialect. Studies of the Chinese Language, 02, 129-138.

[5] Grice, H.P. (1975). Logic and Coversation. In Cole, P. \& Morgan, J., (eds.). Syntax and Semantics :Speech Acts. New York: Academic Press. 41-45.

[6] Han Weiwei. (2013). A Study on the Pronunciation of Chongqing Dialect. MA. dissertation, Tianjin Normal University.

[7] Han Ziman. (2002). On the Limitation of Dialect in Translation. Journal of PLA University of Foreign Languages, 04, 86-90.

[8] Huang Guoying. (1994). The Levels of Particles at the end of Sentences.Studies in Language and Linguistics, 01, 1-9.

[9] Lakoff, G \& Johnson, M. (1980). Metaphors we live by. London: The University of Chicago Press.

[10] Lakoff, G. (1987). Women, Fire, and Dangerous Things. Chicago, IL: University of Chicago Press.

[11] Lakoff , G. (1993). The contemporary theory of metaphor. Cambridge: Cambridge University Press.

[12] Langacker, R. (1987). Foundations of Cognitive Grammar vol.1. Stanford: University of Stanford Press.

[13] Langacker, R. (1991). Foundations of Cognitive Grammar vol.2. Stanford: University of Stanford Press.

[14] Langacker, R. (2001). Discourse in Cognitive Grammar. Cognition Linguistics, 12 (2), 143-188.

[15] Le Meiyun \& Ling Dexiang. (1994). An Analysis of Errors in English Pronunciation of students in Different Families of Chinese Dialect. Foreign Languages Research, 03, 55-60.

[16] Liu Juhong. (1998). The Difficulties and Interference of Tibetan students' society, language and cultural background to the Learning of English. Journal of Southwest China Normal University (Social philosophy Edition), 05, 41-44.

[17] Mao Lingshuang. (2013). An Analysis of the Differences between Chongqing Dialect and Mandarin from the Perspective of Phonetics and Phonology. Intelligence, 09, 212-213.

[18] Ming Maoxiu. (2016). An Experimental Study on the Tone of Chongqing Dialect. Ph.D. dissertation, Southwest University.

[19] Mo Qiyang \& Duan Yun. (2012). A Cognitive Linguistic Study of Speech Act Force. Foreign Languages Research, $03,21-26$.

[20] Mrayat, A. (2015). Negative Particles and Morphemes in Jordanian Arabic Dialects. Journal of Education and Practice, 6, 87-90.

[21] Peng Yongzhao. (1988). Several Modal Words in Chongqing Dialect.Journal of Chongqing Normal University, $02,69-72$.

[22] Ronald,W. (1986). An Introduction to Sociolinguistics. Oxford: Blackwell Publishers Ltd.

[23] Talmy, L. (1988). Force Dynamics in Language and Cognition. Cognitive Science, 12(1), 49-100.

[24] Talmy, L. (2000). Toward a Cognitive Semantics. Cambridge: MIT Press.

[25] Talmy, L. (1995). The Cognitive Culture System. Monist. 78(1), 80-114.

[26] Ungerer, F. \& Schemid, H-J. (1996). An Introduction to Cognitive Linguistics. London: Longman.

[27] Wang Changxue. (1996). On the Sound of Children in Chongqing Dialect. Journal of Southwestern University, 04, 65-67.

[28] Wen Xu. (2014). Cognitive Foundations of Language. Beijing: Science Press.

[29] Zhao Yanfang. (2001). An Introduction to Cognitive Linguistics. Shanghai: Shanghai foreign language education press.

[30] Zhu Dexi. (1983). Metareference and Indirect-reference--The Grammar Function and Semantic Function of "de, zhe, suo, zhi" in Chinese Nominalization. Dialect, 1, 16-31.

Liyao Tang was born in Sichuan, China. She is currently an MA candidate specializing in Cognitive Linguistics at the College of Foreign languages and Literatures, Chongqing Normal University, China. Her interests cover Cognitive Linguistics and Language Teaching.

Lian Xiong was born in Chongqing, China. She is currently an MA candidate specializing in English and American Literature at the College of Foreign languages and Literatures, Chongqing Normal University, China. Her interests cover British Literature and American Literature. 